\title{
Analysis of Near Space Hypersonic Glide Vehicle Trajectory Characteristics and Defense Difficulties
}

\author{
${ }^{1, a}$ Shaoxin Feng, ${ }^{1, b}$ Yasheng Zhang \\ ${ }^{1}$ The Academy of Equipment, Beijing 101416, China; \\ a985464922@qq.com, blizhizys@126.com
}

\begin{abstract}
Keywords: Hypersonic glide vehicle, Skip trajectory characteristics, Kinetic weapon, Defense difficulties

Abstract. The vertical trajectory characteristics of near-space hypersonic glide vehicle are researched in the paper. Firstly the model of 3-free degree is established; for skip-glide trajectory, the influences of initial velocity, height and fight path angle to trajectory are analyzed by the numerical simulation method; difficulties of Kinetic anti-missile weapon in defensing near-space vehicle are summarized. The study can help improve the understanding for hypersonic target characteristics, and provide a reference for trajectory forecasting and the design and development of near-space defense abilities.
\end{abstract}

\section{Introduction}

Hypersonic glide vehicle is generally launched by ground-based rockets or released from the space-based platform into the atmosphere, and flight at hypersonic speed in near space for a long distance relying on its own excellent aerodynamics of lifting body structure [1]. US Hypersonic Technology Vehicle (HTV-2) [2] and Advanced Hypersonic Weapon (AHW) are typical boost-glide vehicles. Table 1 shows some of the typical target characteristics of the near space hypersonic glide vehicle.

Table1 Target Characteristics of Typical Near Space Hypersonic Glide Vehicles

\begin{tabular}{ccc}
\hline Typical Aircraft & AHW & HTV-2 \\
\hline Range/km & $5000-6000$ & 16700 \\
Lateral Motorized Distance $/ \mathrm{km}$ & 2000 & 5560 \\
Gliding Time/s & 1600 & 2400 \\
Average Mach Number & $8-10$ & $14-16$ \\
Maximum Flight Mach Number & $10-15$ & $20-25$ \\
Maximum Overload & 2.5 & 3 \\
RCS $/ \mathrm{m}^{2}$ & $0.01-0.1$ & $0.01-0.1$
\end{tabular}

When it flights in near space and obtains a certain height and speed, the vehicle can still achieve horizontal and vertical Maneuver for a wide range relying on its structural advantages of high lift to drag ratio. It not only overcomes the traditional parabolic ballistic trajectory shortcomings of poor maneuverability, but also has the advantages of long range and strong maneuverability, while compared with the conventional hypersonic cruise missile. With the consensus that near space hypersonic glide vehicles can enrich the connotation and the concept of space operations, challenge and impact on traditional combat mode and has broad prospects for military applications, all countries have attached great importance to the studies on the ballistic characteristics of near space hypersonic glide vehicle. Reference [4] studied the influence of initial height, angle and velocity on ballistic equilibrium gliding state in numerical methods, and analyzes the reasons for the formation of skipping. Reference [5] improved methods to study the ballistic characteristics of two kinds of typical trajectory, the equilibrium gliding and skip gliding. In reference [6] problems of ballistic characteristics and parameter optimization skip hypersonic aircraft are also discussed.

In this paper, a hypersonic glide vehicle gliding reentry ballistic characteristics are analyzed through modeling and simulation methods, and the difficulties of vehicle kinetic energy weapon systems to defense this type vehicle are discussed from the range, speed, altitude, overload, etc. It provides direction for capacity building of near space defense. 


\section{Trajectory Modeling and Simulation}

\subsection{Basic assumptions}

In this paper, a US hypersonic glide vehicle for the study, ignoring the Earth's rotation and perturbation. In fact, the boost phase process of reentry gliding aircraft and ballistic missiles are the same, so only the reentry gliding segment model is established.

\subsection{Dynamics model of reentry gliding stage}

The reentry gliding stage of hypersonic glide vehicle is unpowered, and BTT turning technology is used with the flattened body, so it is assumed that during the flight the sideslip angle is zero. Then the vehicle centroid three degrees of freedom motion equation [7] is as follows:

$$
\left\{\begin{array}{l}
\frac{d r}{d t}=V \sin \gamma \\
\frac{d \theta}{d t}=\frac{V \cos \gamma \sin \psi}{r \cos \phi} \\
\frac{d \phi}{d t}=\frac{V \cos \gamma \cos \psi}{r} \\
\frac{d V}{d t}=-\frac{D}{m}-g \sin \gamma \\
\frac{d \gamma}{d t}=\frac{1}{V}\left[\frac{L}{m} \cos \sigma+\left(\frac{V^{2}}{r}-g\right) \cos \gamma\right] \\
\frac{d \psi}{d t}=\frac{1}{V}\left[\frac{L \sin \sigma}{m \cos \gamma}+\frac{V^{2}}{r} \cos \gamma \sin \psi \tan \phi\right]
\end{array}\right.
$$

Where $r$ is the geocentric distance; $\theta$ is the latitude; $\phi$ is the longitude; $V$ is the speed; $\gamma$ is the trajectory declination angle(the angle between the local horizontal, upwards is positive); $\psi$ is the trajectory inclination angle (the angle between the north direction, right side is positive); $\sigma$ the tilt angle; $\omega$ is the earth rotation angular velocity; $L$ is the lift; $D$ is the drag.

\subsection{Aerodynamic Model and Atmospheric Model}

The lift and drag that aircraft suffers when flying in the atmosphere are calculated as follows:

$$
\left\{\begin{array}{l}
D=\frac{1}{2} \rho V^{2} C_{D} S \\
L=\frac{1}{2} \rho V^{2} C_{L} S
\end{array}\right.
$$

where $\rho$ is the density of the atmosphere; $C_{L}$ and $C_{D}$ are the drag coefficient and lift coefficient; $S$ is the reference area. At hypersonic conditions, lift and drag coefficients change with the angle of attack and Mach number, in references [8], the lift and drag coefficients were fitted approximately as the function of the angle of attack and the speed by the method of non-linear least squares:

$\left\{\begin{array}{l}C_{L}=0.0513 \cdot \partial+0.2945 \cdot \exp (-0.1028 \cdot M a)-0.2317 \\ C_{D}=0.000724 \cdot \partial^{2}+0.406 \cdot \exp (-0.323 \cdot M a)+0.024\end{array}\right.$

where the USSA76 model is used to settle the aerodynamic.

\section{Trajectory simulation and analysis}

\subsection{Simulation condition setting}

Table 1 gives to certain aircraft parameters. Initial conditions for simulation are: initial height $h_{0}=120 \mathrm{~km}$, initial speed $v_{0}=5874 \mathrm{~m} / \mathrm{s}$, initial velocity angle $\gamma=-1.5^{\circ}$, tilt angle $\sigma=0^{\circ}$. End condition is terminal velocity $v_{f}=1000 \mathrm{~m} / \mathrm{s}$. In the initial conditions, variation laws of range, altitude, speed and overload in the launch coordinates are obtained by programming and simulating in $\mathrm{C}++$ software. 
Table 2 aircraft parameters and simulation conditions

\begin{tabular}{ccc}
\hline Variable & Symbol & Value / Unit \\
\hline Mass & $\mathrm{m}$ & $907.20 / \mathrm{kg}$ \\
Aerodynamic Reference Area & $\mathrm{S}$ & $0.4839 / \mathrm{m}^{2}$ \\
\hline
\end{tabular}

\subsection{Range}

Fig. 1 shows the variation of hypersonic glide vehicle range with time in the given initial conditions. As can be seen from the Fig., the range reached $6000 \mathrm{~km}$ when the aircraft glides for 1250 s, which is close to the referenced parameters of the aircraft, what indicating the model meet the requirements; at the same time the characteristics of long flight distance, and high flight speed of the aircraft is reflected. When the aircraft is put into use as a weapon, it can achieve Prompt Global Strike [9] within 1h.

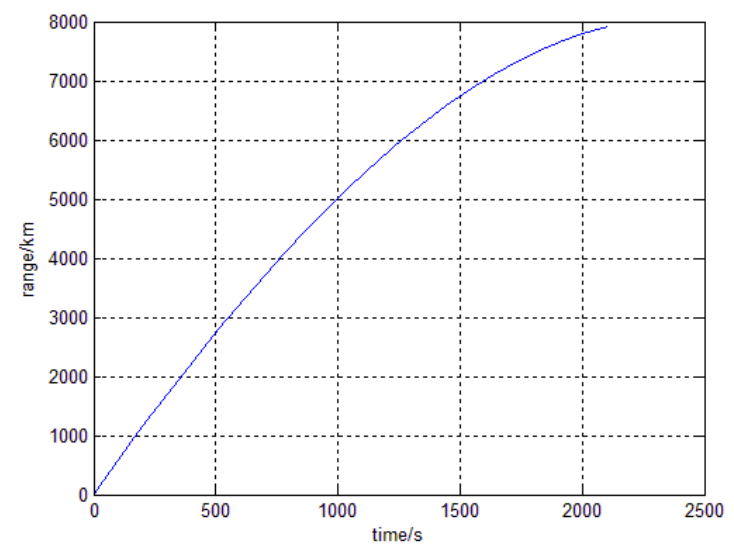

Fig. 1 Range change with time

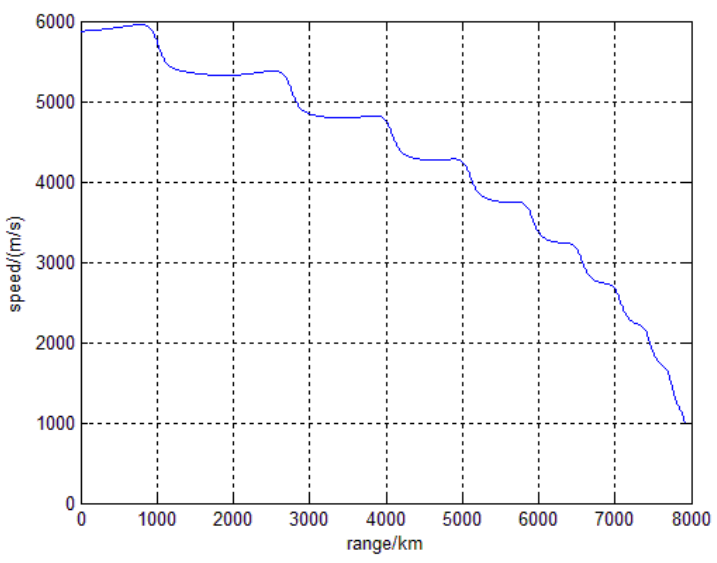

Fig. 2 Speed change with range

\subsection{Speed}

Fig. 2 illustrates the change relationship between speed and range of hypersonic glide vehicle in the given initial conditions. As can be seen from the Fig., speed can still reach $3000 \mathrm{~m} / \mathrm{s}$ or more after the aircraft flying 6000km.Features of high energy combat dive, fast flying and strong aggressive of hypersonic glider aircraft are reflected.

\subsection{Height}

Fig. 3 and 4 represent the change relationships between height and range in the given initial conditions and different initial height conditions. As can be seen from the Fig., the aircraft flight altitude changes in the form of periodically jumping in gliding stage, the peaks and troughs of each cycle are decreasing, the whole gliding stage is above the $30 \mathrm{~km}$, and the higher the initial height, the larger the ballistic jump. The impact of initial height on the range is relatively small. High maneuverability and penetration ability is reflected.

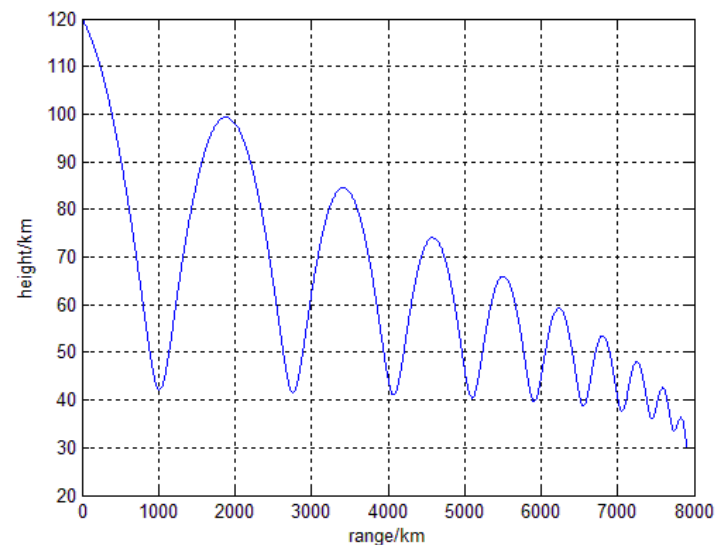

Fig. 3 Height change with range

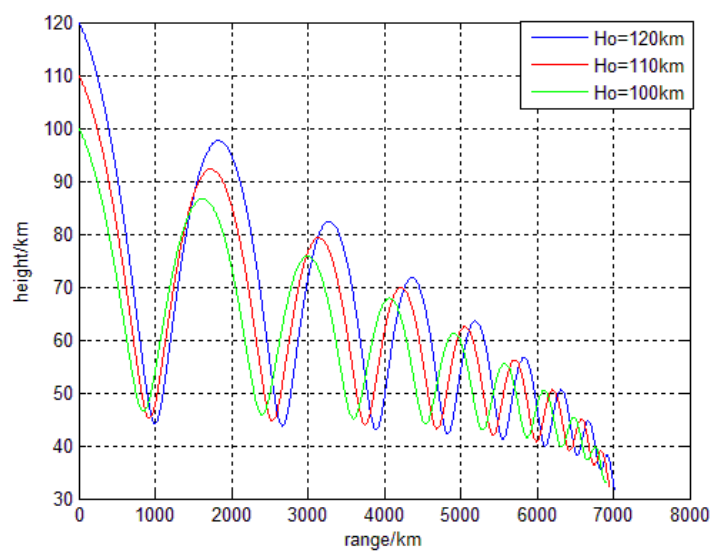

Fig. 4 Height change with range in different initial height conditions

Fig. 5 shows height changes with range in different initial conditions of angle of attack. When the angle of attack increases, you can find decay cycle of height becomes smaller and attenuation 
amplitude becomes large. The impact of the initial angle of attack on range is relatively large. Fig. 6 shows the height variation with range in different condition of initial speeds inclination. As can be seen from the Fig., with the speed of inclination becomes large, attenuation height and period both become smaller, as well as range. Obviously the impact of initial velocity angle on range is relatively large.

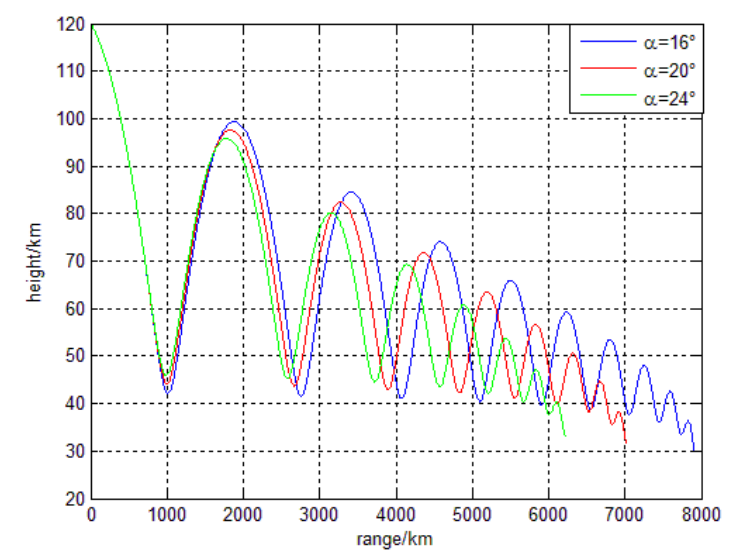

Fig.5 Height change with range in different initial conditions of angle of attack

\subsection{Overload}

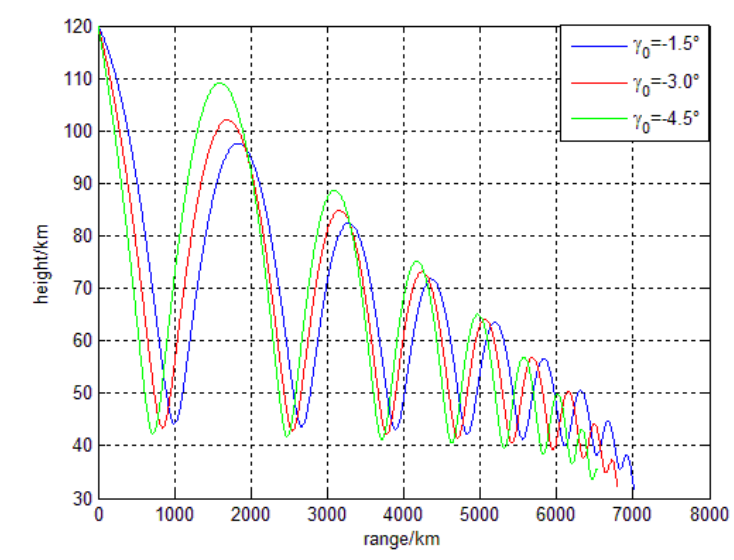

Fig.6 Height variation with range in different condition of initial speeds inclination

Fig. 7 shows relationship between overload and range in the given initial condition. As can be seen from the Fig., the maximum overload of hypersonic glide vehicle is above 4.5. The overload can still reach 3 after the aircraft slides more than $6000 \mathrm{~km}$, which reflects the high maneuverability of hypersonic glide vehicle.

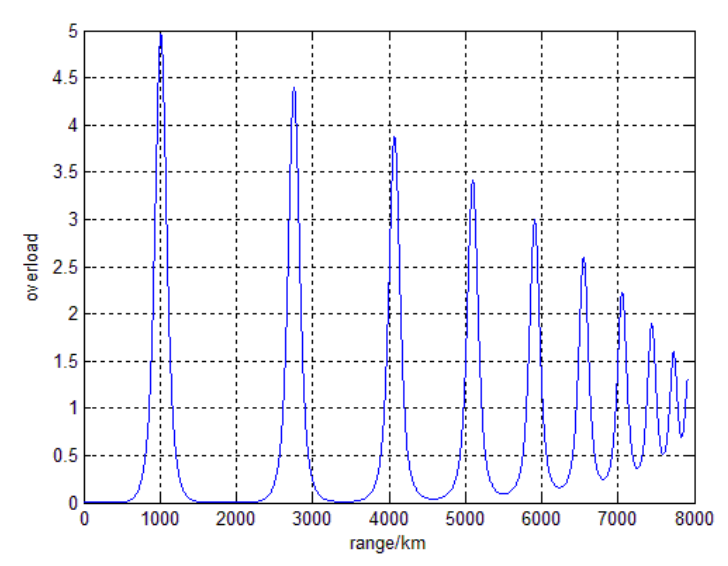

Fig. 7 Overload change with range

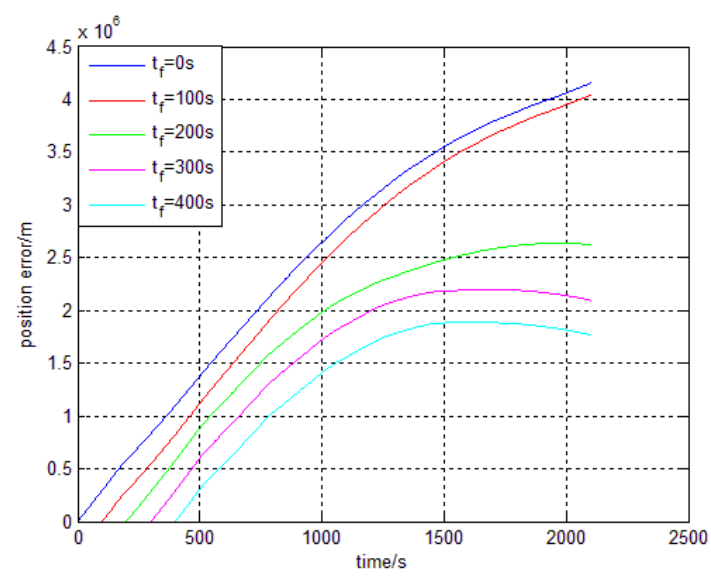

Fig. 8 Position error with time at different time

\subsection{Trajectory position error analysis}

When the early warning and detection equipment obtains target's position and velocity, the kinetic energy system will use the information of time $t$ to predict trajectory of near space hypersonic glide vehicle with the ballistic trajectory pre-forecasting method. Then it will produce a position error. Fig. 8 shows the positions error's change with time at different time information. As can be seen from the Fig., the position error is growing and reaches hundreds of kilometers 100s after predicting time, which is beyond the ability of existing kinetic energy weapons to amend.

\section{Difficulties of defense}

Currently, kinetic energy weapons are widely used in the US missile defense system. Table 3 shows the main tactical indicators of the kinetic energy weapons in existing missile defense system of US [10]. On the basis of analyzing and summarizing the trajectory characters of near space hypersonic glide vehicle, comparing them with the main performance parameters of existing kinetic weapons, the difficulties and deficiencies are obtained when the kinetic defense such a craft. 
Table 3 US main kinetic energy weapons performance indicators

\begin{tabular}{|c|c|c|c|c|c|}
\hline Performance Parameters & KEI & GBI & $\begin{array}{c}\text { SM-3 Block } \\
1 \mathrm{~A} / 1 \mathrm{~B}\end{array}$ & THAAD & PAC-3 \\
\hline $\begin{array}{l}\text { Maximum Operation } \\
\text { Distance (km) }\end{array}$ & - & 5500 & 500 & 300 & 40 \\
\hline $\begin{array}{l}\text { Maximum Operation } \\
\text { Altitude }(\mathrm{km})\end{array}$ & 200 & $>1800$ & 600 & 150 & 45 \\
\hline $\begin{array}{l}\text { Minimum Operation } \\
\text { Altitude }(\mathrm{km})\end{array}$ & 30 & - & 70 & 40 & 15 \\
\hline $\begin{array}{l}\text { Terminal Guidance } \\
\text { Method }\end{array}$ & $\begin{array}{l}\text { Automatic } \\
\text { Guidance } \\
\text { System }\end{array}$ & $\begin{array}{l}\text { Multicolor } \\
\text { Seeker }\end{array}$ & $\begin{array}{l}\text { Passive } \\
\text { Infrared } \\
\text { Seeker }\end{array}$ & $\begin{array}{l}\text { Passive } \\
\text { Infrared } \\
\text { Seeker }\end{array}$ & $\begin{array}{c}\text { C-band } \\
\text { Semi-active } \\
\text { +Ka-band active } \\
\text { Radar }\end{array}$ \\
\hline Maximum Speed (km/s) & 6 & 7.5 & 3-3.5 & 2.5 & 1.7 \\
\hline
\end{tabular}

(1)Limited ability of midcourse and terminal guidance shifting

Kinetic energy weapons of missile defense system are designed to against ballistic missiles, of which the midcourse guides in the mode of pre-forecasting passive segment trajectory of ballistic missile and predicting the hit points. However, due to the effects of dynamic after the near space hypersonic glide vehicle re-entries the atmosphere, its flight altitude and speed vary widely and maneuverability changes strongly, resulting that the mobile trajectory can't be accurately forecast and a distance of hundreds of one thousand meters between kinetic energy weapons and target at the end of the guidance. Although a long-wave infrared seeker is used in "standard -3", whose detection distance range to tactical ballistic missile up to $300 \mathrm{~km}$, the larger missile-target position deviation will lead to the target exceeding its detection angle range of $\pm 1.5^{\circ}$. Eventually the midcourse and terminal guidance shifting can’t be achieved.

\section{(2)Lack of Maneuverability}

Generally, kinetic energy weapons are required to have multiple maneuvering abilities to target when combating. The near space hypersonic glide vehicle's Maneuverability can be up to about 3g, which raised high demands to the kinetic.

\section{(3)Velocity being not enough}

Near space hypersonic glider aircraft still has the speed of $4000 \mathrm{~km} / \mathrm{s}$ after flying for $5000 \mathrm{~km}$. When defensing according to proportional navigation, kinetic weapons must have the speed of at least 1.2 times faster than the target. Generally the height suitable for defensing near space hypersonic glide vehicle is less than $40 \mathrm{~km}$, but kinetic weapon's max speed with this height capability is less than $3.5 \mathrm{~km} / \mathrm{s}$ when it is close to the target. Obviously, the speed for near space hypersonic glide vehicle is not enough.

\section{(4) Lack of defense range}

It can be seen from Table 3, terminal defense system within the atmosphere (such as PAC-3) is capable of defending goals with height range $20-40 \mathrm{~km}$, but the collision probability is very low when hypersonic glide vehicle subducted with a speed of $3000 \mathrm{~m} / \mathrm{s}$, and the defense distance is not enough when defensing targets in the middle of flight. The terminal high-level and mid-defense system's(such as THAAD, SM-3) defense distance can reach $500 \mathrm{~km}$ or more, but the defense height is above $40 \mathrm{~km}$, higher than the aircraft's gliding height in near space; Thus, the existing kinetic energy weapon systems produce defensive blind spot against the near space hypersonic glide vehicle.

\section{(5)Lack of detection and tracking capability}

In order to provide adequate response times to kinetic energy weapon systems and meet the operation needs of effective collision, the effect distance of radar needs to reach more than $1000 \mathrm{~km}$, as the main ground-based detection and tracking equipment. But near space reentry gliding aircraft flying lowly and the height of most of the time is between $30-40 \mathrm{~km}$, the maximum range of phased array radar is $720 \mathrm{~km}$ when only considering the influence of the earth's curvature. Meanwhile, the aircraft may span the radar beam with fast angular rate in radar region, which can lead to radar's insufficient capacity of stable tracking, as well as the high maneuverability and unpredictability. 


\section{Conclusion}

The reentry gliding ballistic characteristics of a hypersonic glide vehicle are analyzed from the range, speed, altitude, overload, etc. through modeling and simulation methods. On this basis, the difficulties and deficiencies of kinetic weapon system to defense such aircraft are discussed. It will help improve the level of awareness of hypersonic vehicle characteristics, and provide a reference to pre-trajectory forecasting, design and improvement of near space defense capabilities.

\section{References}

[1]. XIE Yu, LIU Luhua, TONG Guojian, XU Mingliang. Weaving Maneuver Trajectory Design for Hypersonic Glide Vehicles[J]. Acta Aeronautica et Astronautica Sinica, 2011, 32(12): 2174-2181.

[2]. Jianlin Li. Research of Near Space Hypersonic Vehicle Development[M]. China Astronautic Publishing House, 2012.

[3]. LIU Chao-feng, WANG Gu, ZHANG Zhen-ming, et al. Technical Requirement Analysis of Air Defense System Intercepting Fast Attack Weapons in Near Space [J]. Modern Defense Technology, 2015, 5: 005.

[4]. Chen Xiaoqing, Hong Zhongxi, Liujianxia. Trajectory Characteristics of Hypersonic Gliding Vehicle[J]. Missiles and Space Vehicle, 2011 (2): 5-9.

[5]. LI Guang-hua, ZHANG Hong-bo, TANG Guo-jian. Typical Trajectory Characteristics of Hypersonic Gliding Vehicle[J]. Journal of Astronautics, 2015, 36(4): 397-403.

[6]. HU Zheng-dong, CAO Yuan, ZHANG Shi-feng, et al. Trajectory Performance Analysis and Optimization Design for Hypersonic Skip Vehicle [J]. Journal of Astronautics, 2008, 29(3): 821-825.

[7]. Zhao Hanyuan. Flight Vehicle Reentry Dynamics and Guidance[M]. National University of Defense Technology Press, 1997.

[8]. Duan G, Sun Y, Zhang M, et al. Aerodynamic coefficients models of hypersonic vehicle based on aero database[C]//Pervasive Computing Signal Processing and Applications (PCSPA), 2010 First International Conference on. IEEE, 2010: 1001-1004.

[9]. U.S. Conventional Prompt Global Strike: Issues for 2008 and Beyond [R]. Committee on Conventional Prompt Global Strike Capability, National Research Council, 2008.

[10]. Chen Shitao, Yang Jianjun, Ma Li. Terminal High Altitude Aera Defense System and Operational Disposition [J]. Winged Missiles Journal, 2011 (5): 61-65. 